\title{
Hybrid probabilistic-geometric shaping in optical communication systems
}

\author{
Zhen Qu and Ivan B. Djordjevic \\ Department of Electrical and Computer Engineering \\ University of Arizona \\ Tucson, USA \\ zhenqu@email.arizona.edu
}

\begin{abstract}
We propose a universal distribution matcher applicable to any two-dimensional signal constellation. We experimentally demonstrate that the performance of the proposed 32-ary quadrature amplitude modulation (QAM), based on hybrid probabilistic-geometric shaping, is superior to probabilistically shaped (PS)-32QAM and regular 32QAM, and comparable to PS-64QAM.
\end{abstract}

Keywords - distribution matcher, geometric shaping, probabilistic shaping, QAM.

\section{INTRODUCTION}

Geometric shaping (GS) and probabilistic amplitude shaping (PAS) have been extensively studied to bridge the gap to the Shannon limit [1-4]. GS scheme suffers non-Gray mapping penalty when binary forward error correction (FEC) codes are applied [1]. PAS scheme can only be used for square quadrature amplitude modulation (QAM), which greatly limits its application [2]. Although the performance of PAS-64QAM in principle is able to approach the Shannon limit, its implementation penalty is not clear in experiments.

In this invited paper, we describe a two-dimensional (2D) distribution matcher (DM) [5,6], i.e., arbitrary probabilistic shaping (APS). Generalized pair-wise optimization (GPO) algorithm is applied here to obtain an optimized 32-ary signal constellation, named here opti32QAM, which is enabled by the hybrid PS and GS schemes. In our experiment, we find that the performance of opti-32QAM is comparable to that of the PS-32QAM, and better than regular 32QAM by $0.9 \mathrm{~dB}$, when capacity is $3.33 \mathrm{bits} / \mathrm{symbol}(\mathrm{b} / \mathrm{s})$. In case of $4 \mathrm{~b} / \mathrm{s}$, opti-32QAM outperforms PS-32QAM and regular 32QAM by $0.2 \mathrm{~dB}$ and $0.7 \mathrm{~dB}$, respectively. Additionally, the performance the opti-32QAM is comparable to PAS-64QAM.

\section{UNIVERSAL PS SCHEMES}

Fig. 1 shows the proposed APS-MQAM scheme. The symbols generated by the DM do not yield M-B distribution. The information bits obtained from bit labeling are used to generate the parity-check bits. After the bit-to-symbol mapping, the parity-check bits will be mapped to $N$-ary QAM (NQAM) symbols, where $N$ is the largest power of 2 to contain the MQAM constellation points with the target probabilities of $>(1-R) / M$. Assuming the target probability distribution of the MQAM symbols is $P_{M}(x)$, the distribution of the MQAM symbols after DM is $P_{D}(x)$, and the NQAM probability distribution is $P_{N}(y)=1 / N$, so that the overall relationship among them can be represented as,

$$
P_{M}(x)=\frac{R P_{D}(x) / \log _{2}(M)+(1-R) P_{N}(y) / \log _{2}(N)}{R / \log _{2}(M)+(1-R) \log _{2}(N)}
$$

If $N=M$, we have that $P_{M}(x)=R P_{D}(x)+(1-$ $R) / M$. Therefore, the target distribution of MQAM can also be achieved after the binary FEC coding.

\section{EXPERIMENTAL DEMONSTRATION}

The diagram of the experimental setup is depicted in Fig. 2. The GPO algorithm is used for the generation of the hybrid PS/GS 32QAM (opti-32QAM) [7]. The resulting constellation diagram of the opti-32QAM is shown in the inset of Fig. 2(a). At the transmitter, the binary data is mapped to the symbol sequence via the $2 \mathrm{D}$ DM. After the bit labeling, low-density parity check (LDPC) encoding, and bit-to-symbol mapping, as described in Fig. 1, the digital symbols are pulse-shaped by a $92 \mathrm{G} \mathrm{Sa} / \mathrm{s}$ arbitrary waveform generator (AWG) to generate 40G Baud electrical signals. Such signals are modulated by a polarization multiplexed (PM) I/Q

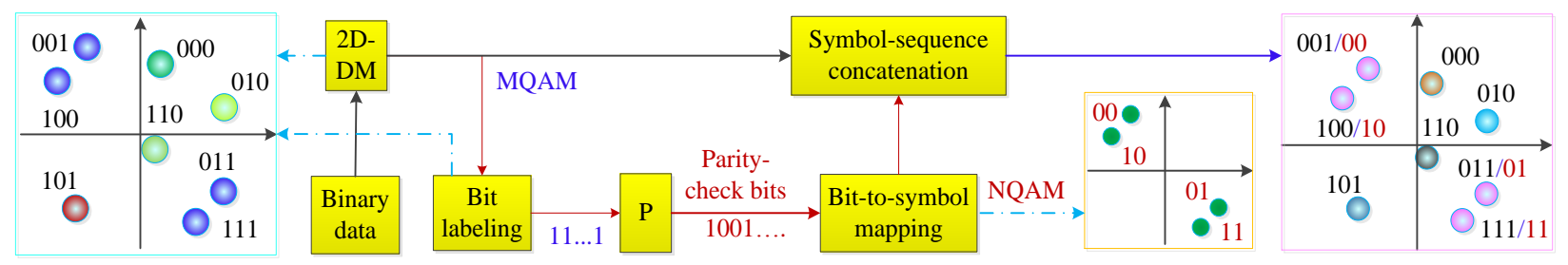

Fig.1. Universal PS schemes based on APS. 

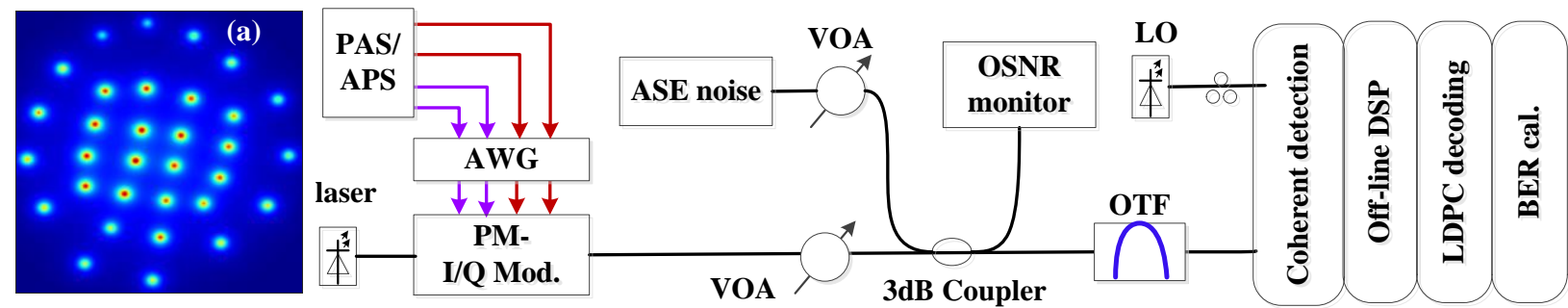

Fig.2. Experimental setup.

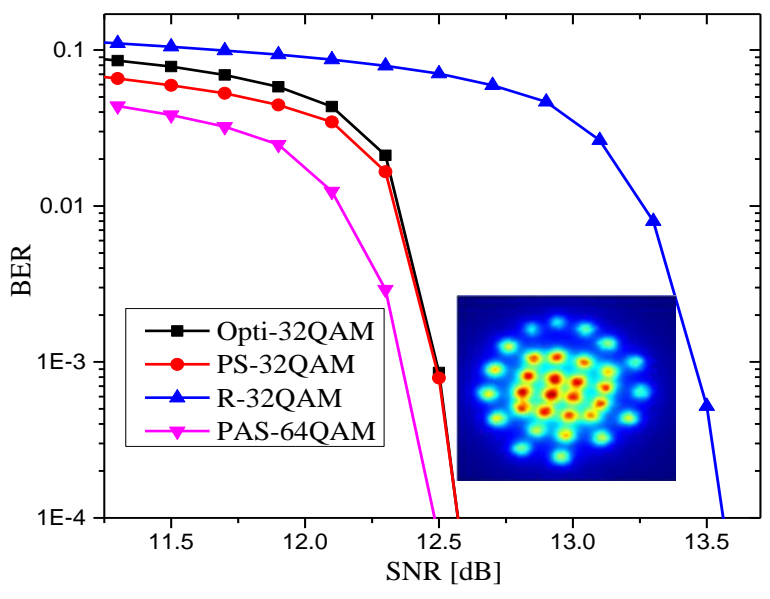

(a)

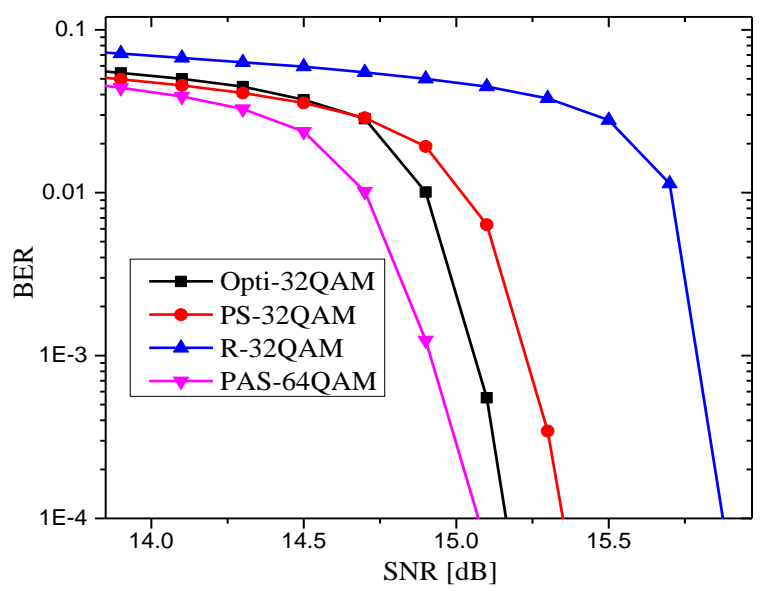

(b)

Fig. 3. The measured post-FEC BER versus SNR for various QAM formats, in cases of: (a) $\mathrm{C}=3.33 \mathrm{~b} / \mathrm{s}$ and (b) $\mathrm{C}=4 \mathrm{~b} / \mathrm{s}$.

modulator, and the resulting 40G Baud PM-MQAM optical signal is mixed with the amplified spontaneous emission (ASE) noise, and filtered out by an optical tunable filter (OTF). After coherent detection, the signals are digitized by a real-time oscilloscope for off-line processing. The LDPC decoder performs the sumproduct algorithm, followed by the bit-error rate (BER) measurements. The DVB-S2 irregular LDPC codes are used for channel coding. For a fair comparison, the performances of the MQAM formats are compared under the same capacity, which is defined as $C=$
$H(p)-m(1-R)$, where $H(p)$ represents the entropy of the PS-MQAM, $m=\log _{2}(M)$, and $R$ is the code rate of the DVB-S2 irregular binary LDPC codes.

Fig. 3 shows the BERs versus SNR performance. When the $C$ is $3.33 \mathrm{~b} / \mathrm{s}$, the performance of opti-32QAM is similar to that of the PS-32QAM, and better than regular 32QAM by $0.9 \mathrm{~dB}$ in the waterfall region. In case of $C=4 \mathrm{~b} / \mathrm{s}$, opt-32QAM outperforms PS-32QAM and regular 32QAM by $0.2 \mathrm{~dB}$ and $0.7 \mathrm{~dB}$, respectively, at the BER of $10^{-4}$. In addition, PAS-64QAM is measured to have less than $0.1 \mathrm{~dB}$ SNR advantage over opti$32 \mathrm{QAM}$ in cases of $C=3.33 \mathrm{~b} / \mathrm{s}$ and $4 \mathrm{~b} / \mathrm{s}$.

\section{CONCLUDING REMARKS}

Two universal DMs have been proposed and used to generate hybrid PS/GS 32QAM and PS-32QAM formats. Our results have shown that the proposed hybrid GS/PS 32QAM could outperform PS-32QAM and regular 32QAM, meanwhile being comparable to PAS-64QAM.

\section{REFERENCES}

[1] Z. Qu and I. B. Djordjevic, "Geometrically shaped 16QAM outperforming probabilistically shaped 16QAM," in European Conference on Optical Communication (ECOC), 2017, paper Th.2.F.4.

[2] G. Böcherer, F. Steiner, and P. Schulte, "Bandwidth efficient and rate-matched low-density parity-check coded modulation," IEEE Trans. Commun., vol. 63, no. 12, pp. 4651-4665, Dec. 2015.

[3] Z. Qu, C. Lin, T. Liu, and I. B. Djordjevic, "Experimental investigation of $\mathrm{GF}\left(3^{2}\right)$ nonbinary LDPC coded non-uniform 9QAM modulation format," in European Conference on Optical Communication (ECOC), 2016, pp. 1112-1114.

[4] Z. Qu, C. Lin, T. Liu, and I. B. Djordjevic, "Experimental study of nonlinearity tolerant modulation formats based on LDPC coded non-uniform signaling," in Optical Fiber Communication Conference (OFC), 2017, paper W1G.7.

[5] Z. Qu, S. Zhang, and I. Djordjevic, "Universal Hybrid Probabilistic-geometric Shaping Based on Two-dimensional Distribution Matchers," in Optical Fiber Communication Conference (OFC), 2018, Paper M4E.4.

[6] S. Zhang, Z. Qu, F. Yaman, E. Mateo, T. Inoue, K. Nakamura, Y. Inada, and I. Djordjevic, "Flex-Rate Transmission using Hybrid Probabilistic and Geometric Shaped 32QAM," in Optical Fiber Communication Conference (OFC), 2018, Paper M1G.3.

[7] S. Zhang, F. Yaman, E. Mateo, T. Inoue, K. Nakamura, and Y. Inada, "A generalized pairwise optimization for designing multidimensional modulation formats," in Optical Fiber Communication Conference (OFC), 2017, paper W4A.6. 